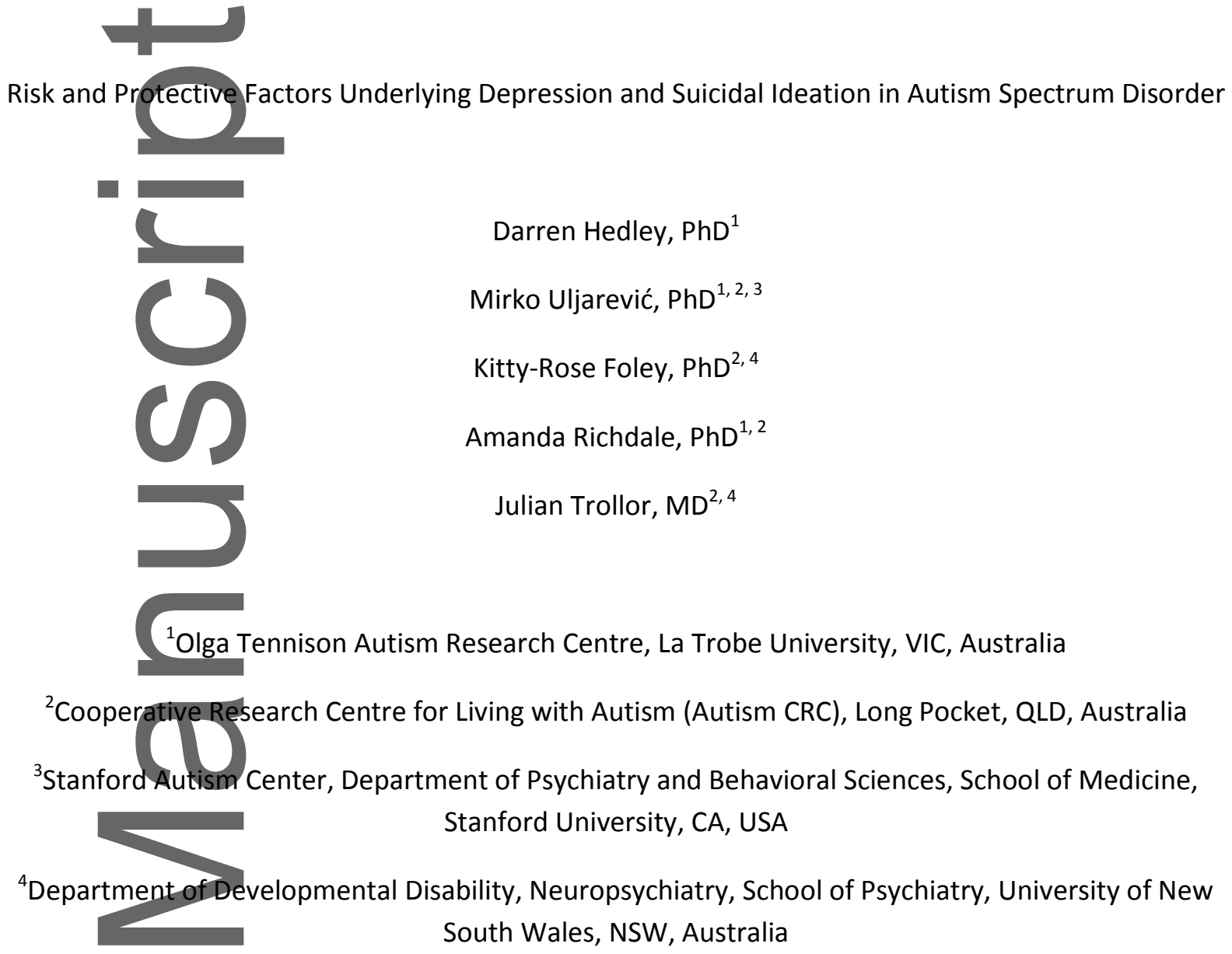

This is truth r manuscript accepted for publication and has undergone full peer review but has not been through tho pyediting, typesetting, pagination and proofreading process, which may lead to differences between this version and the Version of Record. Please cite this article as doi: 10.1002/da.22759.

This article is protected by copyright. All rights reserved. 


\begin{abstract}
Background. People with Autism Spectrum Disorder (ASD) are at significant increased risk of suicidal thoughts and behayior. Given that social difficulties in ASD often lead to social isolation, which can in turn increase the risk for depression, this study examined loneliness and social support as potential risk and protective factors associated with depression and suicidal ideation.

Method. The sample comprised 185 people (92 females) with ASD aged 14 to 80 years who were participating in a national survey.
\end{abstract}

Results. Forty-nine percent of participants returned scores in the clinical range for depression and $36 \%$ reported recent suicidal ideation. Females, comprising almost $50 \%$ of the sample, returned higher depression scores than males, however no differences were identified between males and females in terms of suicidal ideation. Regression analyses revealed that loneliness, satisfaction with social support and ASD traits predicted depression scores. Satisfaction with social support predicted suicidal ideation, however, it was no longer a significant predictor after the effects of depression were taken into account. Path analysis showed that ASD trait severity was independently related to depression, the effect of number of social supports on depression was mediated by loneliness and satisfaction with social support, and that effects of loneliness and satisfaction with social support on suicidal ideation were mediated by depression. The pattern of relationships was nearly identical for males and females.

Conclusions. This study supports a model whereby loneliness and social support operate respectively as protective and risk factors for depression and suicidal ideation in ASD.

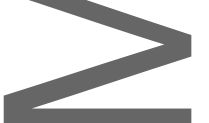

Key Words: Autism spectrum disorder; Depression; Gender; Loneliness; Risk; Social support; Suicidal ideation; Protective factors

Risk and Protective Factors Underlying Depression and Suicidal Ideation in Autism Spectrum

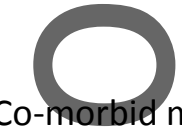
Disorder

Co-morbid mental health conditions are common in individuals with an Autism Spectrum Disorder (ASD) across all age groups (Ghaziuddin, Weidmer-Mikhail, \& Ghaziuddin, 1998; Howlin, 1997; Kim, Szatmari, Bryson, Streiner, \& Wilson, 2000; Lever \& Geurts, 2016; Simonoff et al., 2008), and mood disorders may affect up to half of individuals with ASD at some stage in their life (Hofvander et al., 2009; Wigham, Barton, Parr, \& Rodgers, 2017). Furthermore, people with ASD are at significant risk of suicidal ideation and behavior (Raja, 2014; Segers \& Rawana, 2014) and, although the topic has received some attention in the literature, research in this area is limited (Hedley \& Uljarević, 2018; Zahid \& Upthegrove, 2017). Suicidal ideation in recently diagnosed individuals in the United Kingdom $\left(M_{\text {age }}=31.5, S D=10.9\right.$ years, range $\left.=17-67\right)$ was significantly higher than the general population (odds ratio 9.6, $p<.0001$ ) (Cassidy et al., 2014). Similarly, a Swedish population study identified suicide as the leading cause of premature death in individuals

This article is protected by copyright. All rights reserved.

Corresponding author: Darren Hedley 
with ASD without an intellectual disability (age at death for the study cohort, $M=53.87, S D=24.78$ years), a figure significantly higher than in controls (odds ratio 9.4, $p<.001$ ) (Hirvikoski et al., 2016). These studies highlight the extent and acuteness of the problem. Two recent systematic reviews on suicide in ASD (Hedley \& Uljarević, 2018; Zahid \& Upthegrove; 2017) reported prevalence of suicide attempts from 7 to $47 \%$ and suicidal ideation up to $72 \%$ across child, adolescent, and adult samples. Interventions aimed at reducing suicidal risk in ASD will require a comprehensive understanding of both risk and protective factors (Kleiman \& Liu, 2013). Although research exploring specific risk factors for suicidal behavior in ASD is starting to emerge, the identification of potential protective factors remains largely unexplored (Hedley \& Uljarević, 2018; Segers \& Rawana, 2014; Zahid \& Upthegrove, 2017) It is thus imperative that researchers unravel risk and protective mechanisms underlying increased suicidal risk in this population. In this study we considered both risk and protective factors for depression and suicidal ideation; more specifically, ASD trait severity and loneliness as potential risk, and social support as a potential protective factor.

\section{Autism Spectrum Disorder}

$A S D$ is a lifelong condition characterized by core difficulties in social communication and restricted and repetitive interests or behaviors (American Psychiatric Association, 2013). In individuals with ASD with intellectual functioning (i.e., IQ) in the normative range, it has been argued that ASD symptoms lead to "chronic conflicts, misunderstandings and failures" in personal and vocational relationships, which can lead to the development of comorbid psychiatric conditions including depression and anxiety (Tebartz van Elst, Pick, Biscaldi, Fangmeier, \& Riedel, 2013, p189). According to this framework, the interaction between underlying core symptoms of ASD and environmental demands are thought to underpin the development of co-occurring mental health conditions. Poor social competence coupled with increasing social demands and stress may lead to social withdrawal, which in turn could manifest in depressive symptoms (Tebartz van Elst et al., 2013). There is considerable evidence that people with ASD are susceptible to social isolation, loneliness, and experience reduced availability of social support (Bauminger \& Kasari, 2000; Brugha et al., 2011; Howlin, 2000; Lasgaard, Nielsen, Eriksen, \& Goossens, 2010). Importantly, while isolation and toneliness may increase risk of depression and suicidal ideation or behavior, social support may be a protective factor that is amenable to intervention (Segers \& Rawana, 2014).

\section{Loneliness and social isolation}

Loneliness describes a lack of social contact and the feeling of being cut-off or separated from other people (Hays \& DiMatteo, 1987). Social isolation and loneliness are identified risk factors for depression and suicidal behavior in the general population (Hagerty \& Williams, 1999; Joiner, 1999; 2005; Joinen \& Rudd, 1996; Joiner et al., 2009; Lasgaard, Goossens, \& Elklit, 2011; Mahon, Yarcheski, Varcheski, Cannella, \& Hanks, 2006), but have not received adequate research attention in ASD. In ASD, many individuals experience difficulty establishing and maintaining friendships (Mazurek, 2014), are socially excluded (Brugha et al., 2011; Howlin, 2000), and experience high rates of social isolation and loneliness (Howlin, 2013). As with non-ASD populations, in ASD these factors

This article is protected by copyright. All rights reserved.

Corresponding author: Darren Hedley 
are associated with increased rates of depression, higher anxiety, decreased life satisfaction, and low self-esteem (Mazurek, 2014; Whitehouse, Durkin, Jaquet, \& Ziatas, 2009). Importantly, there is likely to be a bi-directional relationship between loneliness and social relationships as, for example, Mazurek (2014) found greater quantity and higher quality of friendships were associated with decreased loneliness in adults with ASD.

\section{Social support}

Conceptually different from loneliness and social isolation, social support is associated with feeling cared for, loved, and being a member of a larger social network, and can act as a buffer or protective factor for poor mental health outcomes (Cobb, 1976; Kleiman \& Liu, 2013). People with depression tend to report a lack of the availability of others who they can rely upon for support (Winefield, 1979), and greater social support is associated with lower incidence of suicidal ideation (Chioqueta \& Stiles, 2007). There is also evidence that social support acts as a protective factor against suicide attempts in high risk groups (Kleiman \& Liu, 2013) and in the presence of negative or stressful life events (Meadows, Kaslow, Thompson, \& Jurkovic, 2005). In ASD, perceived social support was found to be negatively correlated with loneliness in adolescent boys (Lasgaard et al., 2010) and students with ASD were found to turn to friends for help if they were bullied (Humphrey \& Symes, 2010). However, few studies have examined the protective role of social support in association with depression or suicidal ideation in adults with ASD.

The aim of the present study was to examine loneliness and social support, in addition to the contribution of ASD trait severity, as risk and protective factors associated with depression and suicidal ideation in ASD. We hypothesized that 1 ) loneliness and social support (including the number of people that can be counted on for support and the satisfaction with the support received) would be negatively correlated, 2) greater loneliness would be associated with higher levels of depressive symptoms and suicidal ideation, and 3) greater social support would be negatively associated with depressive symptoms and suicidal ideation. We also hypothesized that 4) greater ASD symptom severity as measured by the AQ-Short (Hoekstra et al., 2011) would be associated with a) higher levels of loneliness, b) lower levels of social support, as well as c) increased levels of depressive symptoms and suicidal ideation. Using path analysis we were interested in determining whether ASD symptom severity would be directly associated with depression and suicidal ideation, or would alternatively reveal an indirect path through the effects on loneliness and social support.

\section{Participants}

\section{Method}

Participants were 195 (94 female) individuals with ASD aged 14-80 years ( $M_{\text {age }}=36.69, S D=$ 15.57 years) who were recruited from a range of sources, including support groups, schools, universities, and advertisements through relevant website and social media. Participants had to report that they received a clinical diagnosis of ASD in order to be included (including any pre DSM-5 diagnostic labels; please refer to Table 1). ${ }^{1}$ They also reported year of diagnosis and type of

This article is protected by copyright. All rights reserved.

Corresponding author: Darren Hedley 
specialist who provided the diagnosis. Self-diagnosed individuals were not included in this study. All participants were required to return a score $>65$ on the AQ-Short; 10 individuals were excluded due to returning a score below this threshold (range 57-65). The final sample included 185 individuals (92 female; $M_{\text {age }}=37.11, S D=15.41$ years). Participant characteristics for males, females and combined, are provided in Table 1. The majority of participants reported a diagnosis of Asperger's syndrome or High Functioning Autism (71.9\%; a further 24.9\% reported a diagnosis of ASD) and had completed some form of post-secondary education. Around one third of participants were living with parents, over one third reported living as a couple and one fifth reported living alone.

Between-group differences for participant characteristics based on gender were examined using Pearson's chilsquare test. The sample was characterized by more male than female secondary students, however there were more females with post-secondary level education. Significant gender differences were also identified for living conditions, with more males living with parents and a higher frequency of females than males living alone. Males and female participants did not differ in terms of employment, diagnostic category, or age.

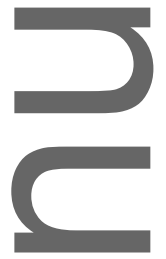

[Enter Table 1 about here]

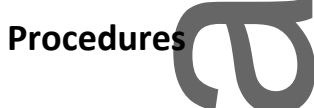

Participants were recruited through the nationwide Australian Longitudinal Study of Adults with Autism (ALSAA; target age range 25 years and older) and the Longitudinal Study of Australian School Leavers with Autism (SASLA; target age range 15-25 years). The research was approved by the University of New South Wales Human Research Ethics Committee HC15001 (ALSAA) and La Trobe University Human Ethics Committee No. 14-095 (SASLA), and informed consent was obtained from all participants. The two studies follow similar recruitment and data collection guidelines but target different age ranges. Recruitment occurs through advertisement and the assistance of ASD specific organizations, online communities, self-advocacy networks, and health professionals. Participants provided demographic information and answered questionnaires online using Qualtrics survey software (Qualtrics, 2017), or paper copies were sent out if requested. Data presented here represent the first data point from the longitudinal studies and therefore a cross-section of the study sample.

Measures

Cronbach's alphas for all instruments for the current study are provided later in Table 3.

Alphas reported in this section are from the instrument validation studies.

This article is protected by copyright. All rights reserved.

Corresponding author: Darren Hedley 
The Abridged Version of the Autism Spectrum Quotient (AQ-Short) (Hoekstra et al., 2011) is a 28 item, self-report measure of ASD traits based on DSM-IV-TR (American Psychiatric Association, 2000) criteria for ASD. The AQ was designed to assess ASD trait severity in individuals with IQ in or above the average range (Baron-Cohen, Wheelwright, Skinner, Martin, \& Clubley, 2001).

Respondents answer statements such as "I prefer to do things the same way over and over again" on a 4-point Likert-type scale, from 1 "definitely agree" to 4 "definitely disagree" (score range 28-112), with higher scores indicating increased ASD trait severity. Using a cut-off $>65$, sensitivity and specificity to ASD is .97 and .82 , respectively, making it a viable screening instrument where formal diagnostic evaluations are not possible. In the present study the AQ-Short was used as a method of confirming diagnosis and measuring ASD trait severity. Cronbach's alpha for the abridged version is $.77-.86$.

\footnotetext{
The University of California Los Angeles Loneliness Scale-Short Form (ULS-8) (Hays \&
} DiMatteo, 1987) is an eight item scale designed to measure feelings of loneliness and social isolation. (n)

Respondents rate how often statements such as "I lack companionship" describe themselves on a 4point Likert-type scale, from 1 "never" to 4 "often" (score range 8-32) with higher scores indicate increased loneliness. Cronbach's alpha $=.84$.

The Social Support Questionnaire-Shortened Version (SSQ-6) (Sarason, Sarason, Shearin, \& Pierce, 1987) is a six item questionnaire designed to measure social support. Respondents' first list the number of people whom they can count on for help or support in certain situations such as "who can you really count on to be dependable when you need help". They then rate how satisfied they are with the support they receive on a 6-point Likert scale from "very satisfied" to "very dissatisfied"'. The questionnaire yields average scores for (a) the number of social supports (SSQ-6N; range 0-9), and (b) satisfaction with the available social support (SSQ-6S; range 1-6), with higher scores indicating greater number or satisfaction with social support. Cronbach's alpha $=.90-.93$.

This article is protected by copyright. All rights reserved. Corresponding author: Darren Hedley 
The Patient Health Questionnaire (PHQ) (Kroenke \& Spitzer, 2002; Kroenke, Spitzer, \& Williams, 2001; Kroenke et al., 2009) is a nine item questionnaire which is designed to detect major and subthreshold depressive disorder and suicidal ideation. Respondents rate how much they have been affected by problems on a 4-point scale from "not at all" to "nearly every day". Items 1 through 8 (range 0-24) provide a reliable measure of depression, while item 9 (range 0-3) provides a useful and independent measure of suicidal ideation (Simon et al., 2013). Higher scores indicate a greater degree of depressive symptoms or increased ideation. Using a cut-off $\geq 10$ (items $1-8$ ), the PHQ has been found to provide reasonable sensitivity (.70-1.00) and specificity (.95-.98) for depressive disorders (Kroenke et al., 2009).

\section{Data cleaning and analysis strategy}

Internal reliability was in the good to excellent range for all scales, and no extreme data points were identified. Tests of normality revealed the scales were not normally distributed and subsequent analyses were performed with 5000 resamples bootstrapping to provide more robust statistics (Efron \& Tibshirani, 1993; Tabachnick \& Fidell, 2007). Correlational analysis was first used to explore the pattern of the relationships between the study variables. To determine whether analyses should be stratified, or whether gender should be entered as a predictor variable, gender effects were examined by (i) running correlational analyses for males and females separately, and (ii) between-group comparisons of relevant study variables. Two regression models were run to identify factors contributing to depression and suicidal ideation, followed by a path analysis to further characterize the nature of the relationship between the study variables. Path analysis was run using AMOS (Version 24; Arbuckle, 2016) software utilizing raw scores for all the variables and the full information maximum likelihood approach. No more than $10 \%$ of missing data per instrument was allowed. As noted, variables were not normally distributed, and data transformation failed toimprove skewness. Path analysis was run with both transformed and untransformed scores, with almost identical findings, untransformed variables are reported here.

\section{Results}

Table 2 provides a summary of depressive symptoms and suicidal ideation derived from PHQ scores. Overall, $48.6 \%$ of the sample returned scores in the clinical range for depression on the PHQ,

This article is protected by copyright. All rights reserved.

Corresponding author: Darren Hedley 
and $35.7 \%$ reported some recent suicidal ideation. Table 3 provides descriptive statistics, correlation and partial correlation coefficients between study variables. Participants reported having an average of just over two people they can count on. We considered the potential impact of being in a relationship on the primary study variables (i.e., AQ-short, loneliness, social support satisfaction and number, depression, suicidal ideation). However, participants who reported they were in a relationship did not differ significantly on these variables when compared to those who were not (all $\left.p^{\prime} s>.05\right)$, with effect sizes ranging from negligible to small ( $\left.d^{\prime} s=.00-.26\right)$. ASD traits assessed by the AQ-Short were positively associated with age, loneliness, and depression, but not suicidal ideation. Loneliness was negatively correlated with social support satisfaction and number. Depression and suicidal ideation were positively correlated with loneliness and negatively correlated with the social support scales. The pattern of results was not affected when controlling for age.
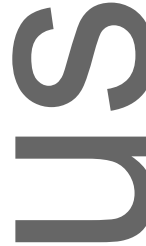

[Enter Table 2 about here]

[Enter Table 3 about here]

\section{Gender effects}

Score distributions and between-group comparisons by gender are provided in Table 4. ASD trait severity was significantly higher for females than males; females also returned significantly higher depression scores. Otherwise, scores for variables of interest, including suicidal ideation, did not differ significantly between males and females. As can be seen in Table 5, correlations between study variables were similar for gender groups as for the whole group, with the exception of suicidal ideation which did not reach a significant correlation with the number of available social supports for males, and the correlation between ASD traits and loneliness not reaching statistical significance for females. Despite these small differences, the direction of effect was identical for both genders, and there were no statistically significant differences in terms of strengths of relationships between genders. Therefore regression and path analyses were run for males and females combined.

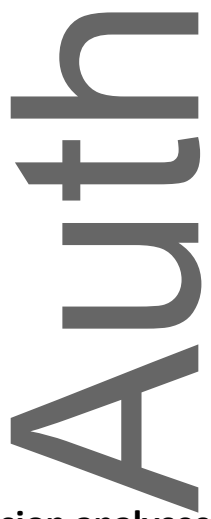

[Enter Table 4 about here]

[Enter Table 5 about here]

Regression analyses

This article is protected by copyright. All rights reserved.

Corresponding author: Darren Hedley 
Full regression models predicting depression and suicidal ideation are presented in Table 6. Using multiple regression, we first examined the predictors of depression. The total model accounted for $29.1 \%$ of variance, $F=18.44, p<.001$, with loneliness $(t=3.11, b=.24, p=.002)$, satisfaction with social support $(t=-2.99, b=-.24, p=.003)$, and ASD traits $(t=3.56, b=.23, p<$ .001 ) emerging as unique predictors of depression scores. Using hierarchical regression, we then examined predictors of suicidal ideation with loneliness and both satisfaction and number of social supports entered into Step 1 and depression and ASD traits entered into Step 2. The first step accounted for $13 \%$ of variance, $F=8.98, p<.001$, with satisfaction with social support emerging as a significant predictor $(t=-3.10,6=-.25, p=.002)$. The second step accounted for an additional $19.9 \%$ of variance $(p<.001)$, with the total model accounting for $32.8 \%$ of variance, $F=17.49, p<$ .001 , with depression $(t=7.24,6=.53, p<.001)$ identified as a unique predictor of suicidal ideation .

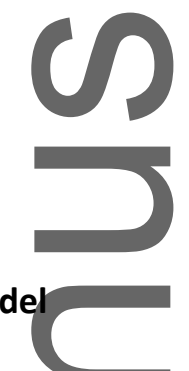

[Enter Table 6 about here]

\section{Path mode}

Figure 1 models the predictive roles that ASD traits, loneliness, the number of social supports, satisfaction with the available social support, and depressive symptoms, play in suicidal ideation for individuals with ASD. The overall fit for the model was excellent $\chi^{2}=2.33, p=.312$; $\mathrm{RMSEA}=.030 ; \mathrm{CFI}=.999, \mathrm{TLI}=.990$. All pathway loadings were in the expected direction. ASD traits predicted less satisfaction with social support $(B=-.13, p=.048)$ and more loneliness $(B=.13, p=$ $.036)$, and it also predicted severity of depression $(\beta=.24, p<.001)$, even after controlling for effects of loneliness and satisfaction with social support. Number of social supports predicted satisfaction with social support $(B=.43, p<.001)$ and loneliness $(B=-.17, p=.009)$, and satisfaction with social support predicted loneliness $(B=-.47, p<.001)$. Both loneliness $(B=.24, p=.002)$ and satisfaction with social support $(B=-.24, p=.002)$ predicted depression. Effects of the number of social supports on depression were mediated by satisfaction with social support $(b=-.43 ; \mathrm{BCa} 95 \% \mathrm{Cl}[-$ $.76,-.15]$, accounting for $45.5 \%$ of the total effect) and loneliness ( $b=-.42 ; \mathrm{BCa}[-.75,-.19]$, accounting for $45.1 \%$ of the total effect). Depression predicted suicidal ideation $(6=.51, p<.001)$. Mediation analyses showed significant effects of both loneliness $(b=.04 ; \mathrm{BCa}[.02, .06])$ and satisfaction with social support ( $b=-.14$; $\mathrm{BCa}[-.21,-.09])$ on suicidal ideation through depression, with depression accounting for $83.9 \%$ and $62.8 \%$ of the total effect, respectively.

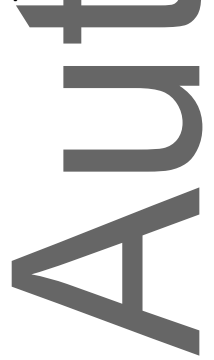

[Enter Figure 1 about here]

\section{Discussion}

This article is protected by copyright. All rights reserved.

Corresponding author: Darren Hedley 
People with ASD have a significantly increased risk of suicidal ideation and behavior when compared to the general population, and suicide is a leading cause of death in this population (Cassidy et al., 2014; Hedley \& Uljarević, 2018; Hirvikoski et al., 2016; Raja, 2014; Segers \& Rawana, 2014; Zahid \& Upthegrove, 2017). However, the factors contributing to such an alarming frequency of suicide risk are not well understood (Hedley \& Uljarević, 2018; Segers \& Rawana, 2014). The present study examined the potential contribution of loneliness and social support to depression and suicidal ideation in people with ASD. In addition, we also explored the contribution of ASD traits on depression and suicidal ideation, both directly and indirectly, through loneliness and social support.

First, this study identified high rates of both depression and suicidal ideation in the study sample, with almost half returning scores in the clinical range for depression, and more than one third reporting recent suicidal ideation. Rates of depression in the present study (48.6\%) were higher than reported in other ASD samples (e.g., 35\%; Cassidy et al., 2014), whereas the rate of suicidal ideation (35.7\%) was lower (e.g., Cassidy et al. reported a rate of 66\%). These differences are most likely accounted for by different methodology and sample characteristics. For example, Cassidy et al. (2014) reported lifetime suicidal ideation and did not directly assess depressive symptoms, instead reporting on the percentage of individuals who had received a diagnosis of depression, thereby potentially underestimating the true rate of depression in the sample. Rates of depression and suicidal ideation in the present study were much higher than in the general population (e.g., 9.5\% for depression and 7.6\% for suicidal ideation; Casey et al., 2008). These results confirm that depression and suicidal ideation are significant problems facing people with ASD (Cassidy et al., 2014; Hirvikoski et al., 2016; Raja, 2014; Segers \& Rawana, 2014; Zahid \& Upthegrove, 2017).

Correlational analysis showed that lower levels of social support, both in terms of the number of social supports and satisfaction with available social support, and higher loneliness, were associated with depression and suicidal ideation. These findings are consistent with non-ASD research where loneliness and social support have been consistently shown to be related to depression and suicide risk (Kleiman \& Liu, 2013; Mahon et al., 2006; Rich \& Bonner, 1987). Although it has been argued that loneliness and social support may increase risk of depression (Lasgaard et al., 2010; Whitehouse et al., 2009), and social isolation and exclusion may increase risk of depression and suicidal behavior in ASD (Cassidy et al., 2014; Gillberg, 2002), as far as the authors are aware this study provides the first explicit exploration of the potential contribution of these factors to suicidal ideation in ASD.

Greater severity of ASD traits were associated with increased loneliness and lower levels of satisfaction with social support, as well as with higher depression severity. Thus, ASD trait severity may present a risk factor for the development and maintenance of psychiatric disturbance (e.g., Tebartz van Elst et al., 2013). It is however important to consider the possibility that greater insight into their condition leads individuals to self-report higher scores on the AQ. This insight may in turn be associated with increased susceptibility to depression. Thus, degree of insight and self-awareness

This article is protected by copyright. All rights reserved.

Corresponding author: Darren Hedley 
may be important for understanding the development of mental health conditions in people with ASD, and the AQ may capture this awareness, even though individuals might not actually be more severely impaired in terms of social and communication abilities compared to peers who return lower scores on the AQ. This is supported by the findings that in ASD, self-awareness is positively associated with depression (Hedley \& Young, 2006; Wing, 1981); IQ is associated with awareness of social limitations (Huang et al., 2017); and depressed adults with ASD were less impaired (i.e., less severely affected) on the ADOS communication and social domains than non-depressed adults (Sterling, Dawson, Estes, \& Greenson, 2008). Furthermore, mental health symptoms may simply be more difficult to assess in those individuals who are more severely affected. The current sample was characterized by a wide spectrum of ASD trait severity on the AQ-Short. It will be important in future to determine the contributions of level of functioning, presence or absence of intellectual disability, and level of awareness, insight, and the ability to articulate these experiences.

Path analysis revealed a number of important findings. Firstly, ASD trait severity independently predicted depression, even after controlling for the effects of satisfaction with social support and loneliness. However, ASD trait severity was not significantly correlated with suicidal ideation. The path from number of social supports to both satisfaction with social support and loneliness was significant, and so was the path from satisfaction with social support to loneliness. The number of social supports did not directly predict depression but the effect was mediated by lower satisfaction and higher loneliness. Finally, paths from both higher loneliness and lower satisfaction with social supports to depression were significant, and their effects on suicidal ideation were mediated by levels of depression. Therefore, while it makes sense to target depression in terms of reducing risk of suicidal ideation and behavior in people with ASD, our findings also indicate that other factors that are either directly (i.e., ASD traits) or indirectly (loneliness, availability and quality of social support) associated with having a diagnosis of ASD may contribute to risk. Thus, it may be beneficial for interventions to assist individuals to identify and increase the availability of quality social support networks, and to provide opportunity for regular social interaction, to minimize potentially harmful effects of social isolation.

Limitations

Ou findings need to be interpreted in light of several limitations. First, due to the higher than usual number of female participants, this study is not representative of the ASD population (e.g., Loomes, Hull, \& Mandy, 2017). Although no specific gender effects were found in this study, it will be important for future research to determine how gender and psychosocial variables interact to predict depression and suicidal behavior. Next, as a cross-sectional study we cannot make claims about causality. We were also limited in our ability to independently confirm diagnosis or assess cognitive level. To address this limitation, we only included participants who met cut-off criteria on the AQ-Short, which exhibits sound psychometric properties when compared to more comprehensive diagnostic measures (Hoekstra et al., 2011), and we reported information on educational attainment. Nonetheless, our study does not offer insights into how the study variables are associated in individuals with ASD and intellectual disability. To understand how ASD severity,

This article is protected by copyright. All rights reserved.

Corresponding author: Darren Hedley 
and also intellectual disability, interact with psychosocial variables, both a broader sample of adults with ASD, and a more in depth assessment of ASD symptoms than is provided by the AQ-Short, is needed.

It is widely acknowledged that there is a lack of validated instruments for the assessment of mental health problems in the ASD population, and this gap is particularly acute in relation to depression (Uljarević et al., 2017; Wigham et al., 2017). We selected measures that have a strong evidende-base in non-ASD clinical and general populations. Although these measures performed well psychometrically in this study, the use of brief assessment measures, which have not been independently validated in people with ASD, nevertheless needs to be considered. More research concerning the reliability of self-report measures to assess states such as loneliness, depression, social supports, and suicidal ideation in individuals with ASD is needed. Future research will also have to explore in more detail the potential impact of ASD severity and level of functioning on the validity of self-report measures. Additionally, it is necessary to consider how people with ASD conceptualize the above-discussed constructs, and whether they may differ from people without ASD. Interestingly, participants who reported being in a relationship did not differ on the primary study variables when compared to those not in a relationship. Future research would benefit from closer examination of the role of intimate personal relationships on the mental health and wellbeing of individuals with ASD.

Although single item measures of suicidal ideation have been found to provide valid and reliable data across other studies (Casey et al., 2008; Simon et al., 2013; Uebelacker, German, Gaudiano, \& Miller, 2011), it will be important for future studies to consider the application of more extensive and targeted assessment of suicidal ideation and behavior. Ideally, this would include a clinical interview (Segers \& Rawana, 2014). Finally, the current study evaluated suicidal ideation, not suicide attempts. Research that examines factors that contribute to suicide attempts in this population is needed.

\section{Conclusions}
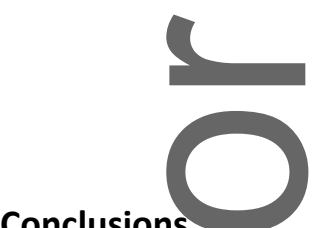

Acknowledging its limitations, our study nevertheless provides the first evidence for a model whereby loneliness and social support operate respectively as protective and risk factors for depression and suicidal ideation in adolescents and adults with ASD. These findings suggest social support and social isolation as viable intervention targets in reducing risk for depression and suicidal ideation in ASD.

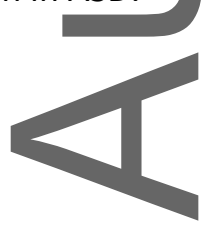

This article is protected by copyright. All rights reserved.

Corresponding author: Darren Hedley 
Footnotes

${ }^{1}$ Consistent with DSM-5 (American Psychiatric Association, 2013) terminology, we use the umbrella term "ASD" to describe participants. However, it is important to note that many participants reported diagnoses consistent with DSM-IV (American Psychiatric Association, 2000) diagnostic terminology

\section{Acknowledgements}

We thank the individuals who participated in this study. The authors acknowledge the financial support of the Cooperative Research Centre for Living with Autism (Autism CRC), established and supported under the Australian Government's Cooperative Research Centres Program. Darren Hedley and Mirko Uljarević share first authorship. Amanda Richdale and Julian Trollor share senior authorship.

\section{Contributors}

$\mathrm{DH}$ completed the literature review. $\mathrm{DH}$ and $\mathrm{MU}$ conceived of the report and prepared the tables. AR and MU selected the study questionnaires. MU and K-R F collected the data. DH and MU completed the data analysis and interpretation. DH and MU wrote the report and JT critically revised the report. All authors reviewed and approved the final submitted version.

\section{Declaration of interests}

DH was supported by funding from DXC Technology and the Australian Government Department of Human Services and Department of Defence. MU and K-R F were each supported by Autism CRC postdoctoral fellowships. The authors declare no other actual or potential conflicts of interest.

\section{Role of funding source}

The funders had no role in the study design, data collection, analysis, data interpretation, or writing of the report. The corresponding author had full access to all the data in the study and final responsibility for the decision to submit the report for publication.

\section{Ethical approval}

The research was approved by the University of New South Wales Human Research Ethics Committee HC15001 (ALSAA) and La Trobe University Human Ethics Committee No. 14-095 (SLLS). All procedures performed in studies involving human participants were in accordance with the ethical standards of the institutional and/or national research committee and with the 1964 Helsinki declaration and its later amendments or comparable ethical standards. Informed consent was obtained from participants after the nature of the study was explained.

This article is protected by copyright. All rights reserved.

Corresponding author: Darren Hedley 


\section{References}

American Psychiatric Association. (2000). Diagnostic and statistical manual of mental disorders $\left(4^{\text {th }}\right.$ edn text rev.). Washington, DC: American Psychiatric Publishing.

American Psychiatric Association. (2013). Diagnostic and statistical manual of mental disorders (5th ed. WWashington, DC: American Psychiatric Publishing.

Arbuckle, J. L. (2016). Amos (version 24.0) [computer program]. Chicago: IBM SPSS.

Baron-Cohen, S., Wheelwright, S., Skinner, R., Martin, J., \& Clubley, E. (2001). The autism spectrum quotient (AQ): Evidence from Asperger syndrome/high functioning autism, males and fernales, scientists and mathematicians. J Autism Dev Disord, 31, 5-17.

Bauminger, N., \& Kasari, C. (2000). Loneliness and friendship in high-functioning children with autism. Child Dev, 71, 447-456.

Brugha, T. S., McManus, S., Bankart, J., Scott, F., Purdon, S., Smith, J., . . Meltzer, H. (2011). Epidemiology of autism spectrum disorders in adults in the community in England. Arch Gen Psychiatry, 68, 459-465.

Casey, P., Dunn, G., Kelly, B. D., Lehtinen, V., Dalgard, O. S., Dowrick, C., \& Ayuso-Mateos, J. L. (2008). The prevalence of suicidal ideation in the general population: Results from the Outcome of Depression International Network (ODIN) study. Soc Psychiatry Psychiatr Epidemiol, 43, 299304.

Cassidy, S., Bradley, P., Robinson, J., Allison, C., McHugh, M., \& Baron-Cohen, S. (2014). Suicidal ideation and suicide plans or attempts in adults with Asperger's syndrome attending a specialist diagnostic clinic: A clinical cohort study. The Lancet Psychiatry, 1, 142-147.

Chioqueta, A.P., \& Stiles, T. C. (2007). The relationship between psychological buffers, hopelessness, and suicidal ideation: Identification of protective factors. Crisis, 28, 67-73.

Cobb, S. (1976). Social support as a moderator of life stress. Psychosomatic Medicine, 38, 300-314.

Efron, B., \& Tibshirani, R. (1993). An Introduction to the Bootstrap. Boca Raton, FL: Chapman \& Hall/CRC.

Ghaziuddin, M., Weidmer-Mikhail, E., \& Ghaziuddin, N. (1998). Comorbidity of Asperger syndrome: A preliminary report. J Intellect Disabil Res, 42, 279-283.

Gillberg, C. (2002). A guide to Asperger syndrome. Cambridge: Cambridge University Press.

This article is protected by copyright. All rights reserved.

Corresponding author: Darren Hedley 
Gotham, K., Unruh, K., \& Lord, C. (2015). Depression and its measurement in verbal adolescents and adults with autism spectrum disorder. Autism, 19, 491-504.

Hagerty, B. M., \& Williams, R. A. (1999). The effects of sense of belonging, social support, conflict, and loneliness on depression. Nurs Res, 48, 215-219.

Hays, R. D., \& DiMatteo, M. R. (1987). A short-form measure of loneliness. J Pers Assess, 51, 69-81.

Hedley, D. \& Uljarević, M. (2018). Systematic review of suicide in Autism Spectrum Disorder: Current trends and implications. Current Developmental Disorders Reports, 5, 65-76.

Hedley, D., \& Young, R. (2006). Social comparison processes and depressive symptoms in children and adolescents with Asperger syndrome. Autism, 10, 139-153.

Hirvikoski, T., Mittendorfer-Rutz, E., Boman, M., Larsson, H., Lichtenstein, P., \& Bölte, S. (2016). Premature mortality in autism spectrum disorder. Br J Psychiatry, 208, 232-238.

Hoekstra, R. A., Vinkhuyzen, A. A., Wheelwright, S., Bartels, M., Boomsma, D. I., Baron-Cohen, S., . . van der Sluis, S. (2011). The construction and validation of an abridged version of the autismspectrum quotient (AQ-Short). J Autism Dev Disord, 41, 589-596.

Hofvander, B., Delorme, R., Chaste, P., Nydén, A., Wentz, E., Ståhlberg, O., . . Leboyer, M. (2009). Psychiatric and psychosocial problems in adults with normal-intelligence autism spectrum disorders. BMC Psychiatry, 9, 1-9.

Howlin, R. (1997). Psychiatric disturbances in adulthood. In P. Howlin (Ed.), Autism: Preparing for Adulthood (pp. 216-235). London: Routledge.

Howlin, P. (2000). Outcome in adult life for more able individuals with autism or Asperger syndrome. Autism, 4, 63-83.

Howlin, P. (2013). Social disadvantage and exclusion: Adults with Autism lag far behind in employment prospects. J Am Acad Child Adolesc Psychiatry, 52, 897-899.

Huang, A. X., Hughes, T. L., Sutton, L. R., Lawrence, M., Chen, X., Ji, Z., \& Zeleke, W. (2017). Understanding the self in individuals with Autism Spectrum Disorders (ASD): A review of literature. Front Psychol, 8, 1422.

Humphrey, N., \& Symes, W. (2010). Responses to bullying and use of social support among pupils with autism spectrum disorders (ASDs) in mainstream schools: A qualitative study. Journal of Research in Special Educational Needs, 10, 82-90.

Joiner, T. E. (1999). A test of interpersonal theory of depression in youth psychiatric inpatients. J Abnorm Child Psychol, 27, 77-85.

Joiner T. E. (2005). Why people die by suicide. Cambridge, MA: Harvard University Press.

This article is protected by copyright. All rights reserved.

Corresponding author: Darren Hedley 
Joiner, T. E., Van Order, K. A., Witte, T. K., Selby, E. A., Ribeiro, J. D., Lewis, R., \& Rudd, M. D. (2009). Main predictors of the Interpersonal-Psychological Theory of Suicidal Behavior: Empirical tests in two samples of young adults. J Abnorm Psychol, 118, 634-646.

Joiner, T. E., \& Rudd, M. D. (1996). Disentangling the interrelations between hopelessness, loneliness, and suicidal ideation. Suicide Life Threat Behav, 26, 19-26.

Kim, J. A. Szatmari, P., Bryson, S. E., Streiner, D. L., \& Wilson, F. J. (2000). The prevalence of anxiety and mood problems among children with Autism and Asperger syndrome. Autism, 4, 117132.

Kleiman, E. M., \& Liu, R. T. (2013). Social support as a protective factor in suicide: Findings from two nationally representative samples. J Affect Disord, 150, 540-545.

Kroenke, K., \& Spitzer, R. L. (2002). The PHQ-9: A new depression diagnostic and severity measure. Psychiatric Annals, 32, 509-515.

Kroenke, K , Spitzer, R. L., \& Williams, J. B. (2001). The PHQ-9: Validity of a brief depression severity measure. JGen Intern Med, 16, 606-613.

Kroenke, $K_{\bar{z}}$ Strine, T. W., Spitzer, R. L., Williams, J. B., Berry, J. T., \& Mokdad, A. H. (2009). The PHQ8 as a measure of current depression in the general population. J Affect Disord, 114, 163173

Lasgaard, M., Goossens, L., \& Elklit, A. (2011). Loneliness, depressive symptomatology, and suicide ideation in adolescence: cross-sectional and longitudinal analyses. J Abnorm Child Psychol, $39,137-150$.

Lasgaard, M., Nielsen, A., Eriksen, M. E., \& Goossens, L. (2010). Loneliness and social support in adolescent boys with Autism Spectrum Disorders. J Autism Dev Disord, 40, 218-226.

Lever, A. G., \& Geurts, H. M. (2016). Psychiatric co-occurring symptoms and disorders in young, middle-aged, and older adults with Autism Spectrum Disorder. J Autism Dev Disord, 46, 1916-1930.

Loomes, R., Hull, L., \& Mandy, W. P. L. (2017). What is the male-to-female ratio in Autism Spectrum Disorder? A systematic review and meta-analysis. J Am Acad Child Adolesc Psychiatry, 56, 466-474.

Mahon, N. E., Yarcheski, A., Yarcheski, T. J., Cannella, B. L., \& Hanks, M. M. (2006). A meta-analytic study of predictors for loneliness during adolescence. Nurs Res, 55, 308-315.

Mazurek, M. O. (2014). Loneliness, friendship, and well-being in adults with autism spectrum disorders. Autism, 18, 223-232.

This article is protected by copyright. All rights reserved.

Corresponding author: Darren Hedley 
Meadows, L. A., Kaslow, N. J., Thompson, M. P., \& Jurkovic, G. J. (2005). Protective factors against suicide attempt risk among African American women experiencing intimate partner violence. Am J Community Psychol, 36, 109-121.

Qualtrics. (2017). Qualtrics (version 2.16) [computer program]. Provo, UT: Qualtrics.

Raja, M. (2014). Suicide risk in adults with Asperger's syndrome. The Lancet Psychiatry, 1, 99-101.

Rich, A. R., \& Bonner, R. L. (1987). Concurrent validity of a stress-vulnerability model of suicidal ideation and behavior: A follow-up study. Suicide Life Threat Behav, 17, 265-270.

Sarason, I. G., Sarason, B. R., Shearin, E. N., \& Pierce, G. R. (1987). A brief measure of social support: Practical and theoretical implications. Journal of Social and Personal Relationships, 4, 497510

Segers, M., \& Rawana, J. (2014). What do we know about suicidality in Autism Spectrum Disorders? A systematic review. Autism Research, 7, 507-521.

Simon, G. E., Rutter, C. M., Peterson, D., Oliver, M., Whiteside, U., Operskalski, B., \& Ludman, E. J. (2013). Do PHQ depression questionnaires completed during outpatient visits predict subsequent suicide attempt or suicide death? Psychiatr Serv, 64, 1195-1202.

Simonoff, E., Pickles, A., Charman, T., Chandler, S., Loucas, T., \& Baird, G. (2008). Psychiatric disorders in children With Autism Spectrum Disorders: Prevalence, comorbidity, and associated factors in a population-derived sample. J Am Acad Child Adolesc Psychiatry, 47, 921-929.

Sterling, L, Dawson, G., Estes, A., \& Greenson, J. (2008). Characteristics associated with presence of depressive symptoms in adults with Autism Spectrum Disorder. J Autism Dev Disord, 38, 1011-1018.

Tabachnick, B. G., \& Fidell, L. S. (2007). Using Multivariate Statistics (5th ed.). Needham Heights, MA: Allyn \& Bacon.

Tebartz van Elst, L., Pick, M., Biscaldi, M., Fangmeier, T., \& Riedel, A. (2013). High-functioning autism spectrum disorder as a basic disorder in adult psychiatry and psychotherapy:

Psychopathological presentation, clinical relevance and therapeutic concepts. Eur Arch Psychiatry Clin Neurosci, 263, 189-196.

Uebelacker, L. A., German, N. M., Gaudiano, B. A., \& Miller, I. W. (2011). Patient Health Questionnaire Depression Scale as a suicide screening instrument in depressed primary care patients: A cross-sectional study. Prim Care Companion CNS Disord, 13, e1-e6.

Uljarević, MM., Richdale, A. L., McConachie, H., Hedley, D., Cai, R. Y., Merrick, H., Parr, J. R., \& Le Couteour, A. (2017). The Hospital Anxiety and Depression scale: Factor structure and

This article is protected by copyright. All rights reserved.

Corresponding author: Darren Hedley 
psychometric properties in older adolescents and young adults with autism spectrum disorder. Autism Research, 11, 258-269.

Whitehouse, A. J. O., Durkin, K., Jaquet, E., \& Ziatas, K. (2009). Friendship, loneliness and depression in adolescents with Asperger's Syndrome. J Adolesc, 32, 309-322.

Wigham, S, Barton, S., Parr, J. R., \& Rodgers, J. (2017). A systematic review of the rates of depression in children and adults with high-functioning Autism Spectrum Disorder. Journal of Mental Health Research in Intellectual Disabilities, 1-21.

Winefield, H.R. (1979). Social support and the social environment of depressed and normal women. Aust N Z J Psychiatry, 13, 335-339.

Wing, L. (1981). Asperger's syndrome: a clinical account. Psychol Med, 11, 115-129.

Zahid, S., \& Upthegrove, R. (2017). Suicidality in Autistic Spectrum Disorders: A systematic review. Crisis, 38, 237-246.

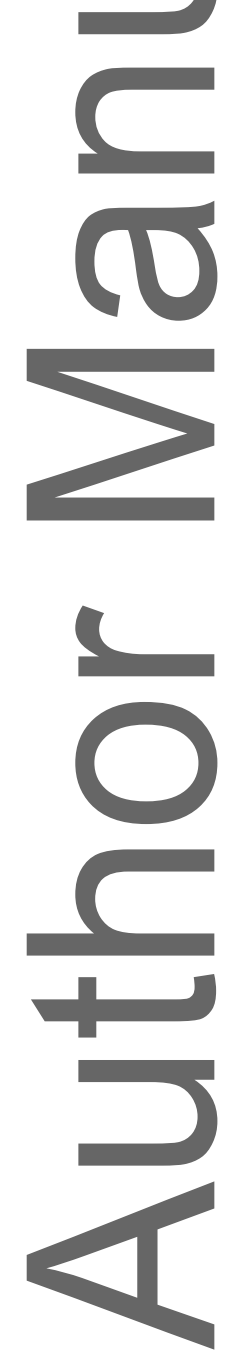

This article is protected by copyright. All rights reserved.

Corresponding author: Darren Hedley 
Table 1. Participant characteristics by gender and between-group gender differences

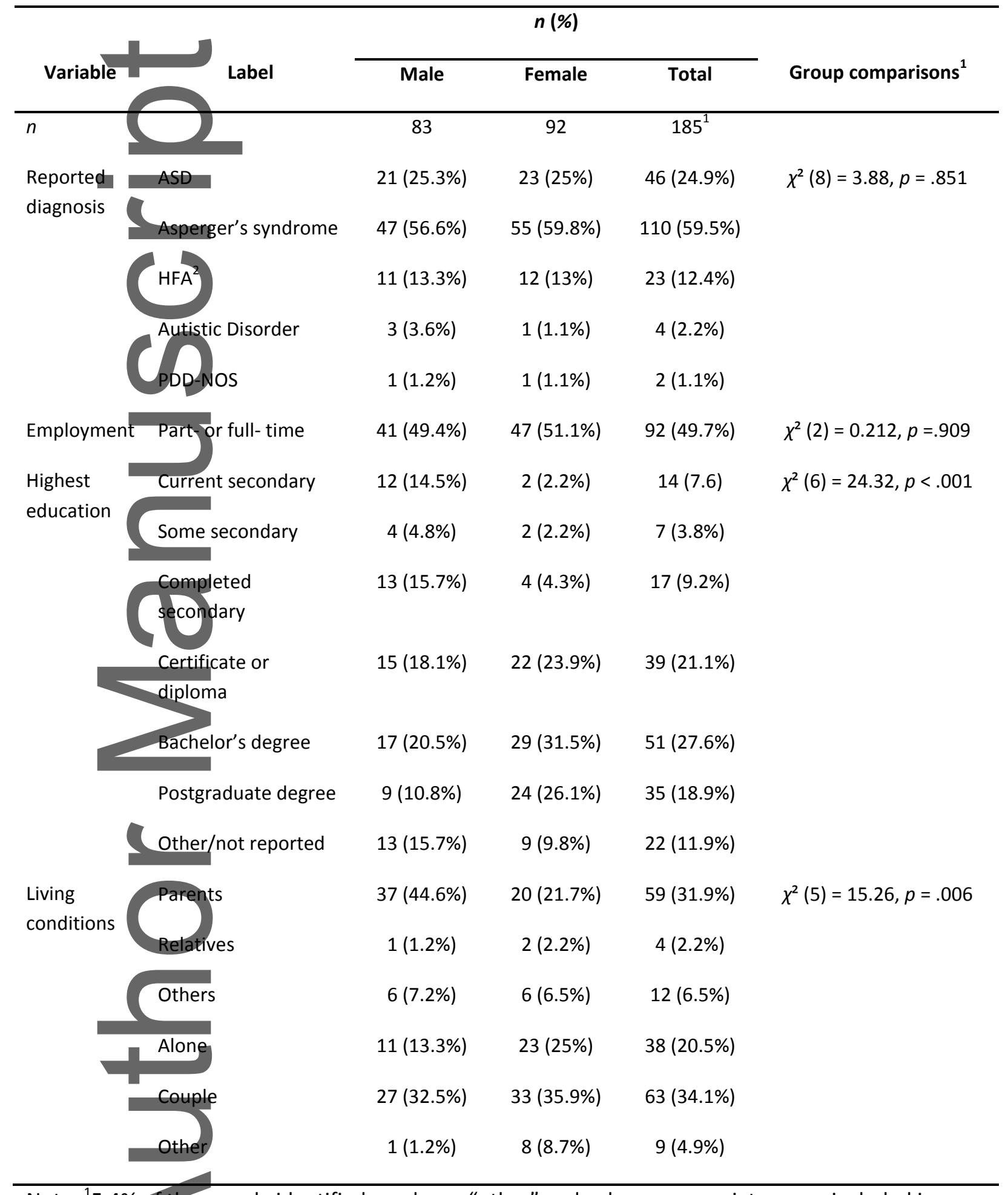

Note. ${ }^{1} 5.4 \%$ of the sample identified gender as "other" and, where appropriate, were included in total and comparison statistics. ${ }^{2}$ Although "High Functioning Autism" (HFA) is not a formal ASD diagnosis, some individuals with an ASD diagnosis with an Intelligence Quotient (IQ) in the normal

This article is protected by copyright. All rights reserved.

Corresponding author: Darren Hedley 
range may identify diagnostically as HFA, and therefore this category was included in the survey. All participants included in this study answered a separate question confirming they had received a formal diagnosis of ASD.

Table 2. Distribution of PHQ depression symptom severity, depression cut-off ( $\geq 10)$, and suicidal ideation $(N=185)$

\begin{tabular}{|c|c|c|}
\hline \multirow{2}{*}{ PHQ } & \multicolumn{2}{|c|}{ Frequency } \\
\hline & $n$ & $\%$ \\
\hline \multirow[t]{5}{*}{- Minimal (0-4) } & 46 & 24.9 \\
\hline & 49 & 26.5 \\
\hline & 47 & 25.4 \\
\hline & 29 & 15.7 \\
\hline & 14 & 7.6 \\
\hline \multirow[t]{2}{*}{ Clinical cut-off ${ }^{1} \quad$ Below $(<10)$} & 95 & 51.4 \\
\hline & 90 & 48.6 \\
\hline Ideation $^{2}$ & 119 & 64.3 \\
\hline al days & 46 & 24.9 \\
\hline han half the days & 11 & 5.9 \\
\hline Nearly every day & 9 & 4.9 \\
\hline
\end{tabular}

Note. ${ }^{1}$ Items 1-8. ${ }^{2}$ Item 9.

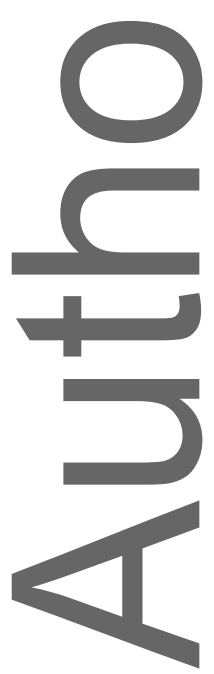

This article is protected by copyright. All rights reserved.

Corresponding author: Darren Hedley 
Table 3. Study variables (internal reliability, $M, S D$, range) with Pearson's bootstrapped correlations (top), and partial correlations (bottom) controlling for age $(n=185)$

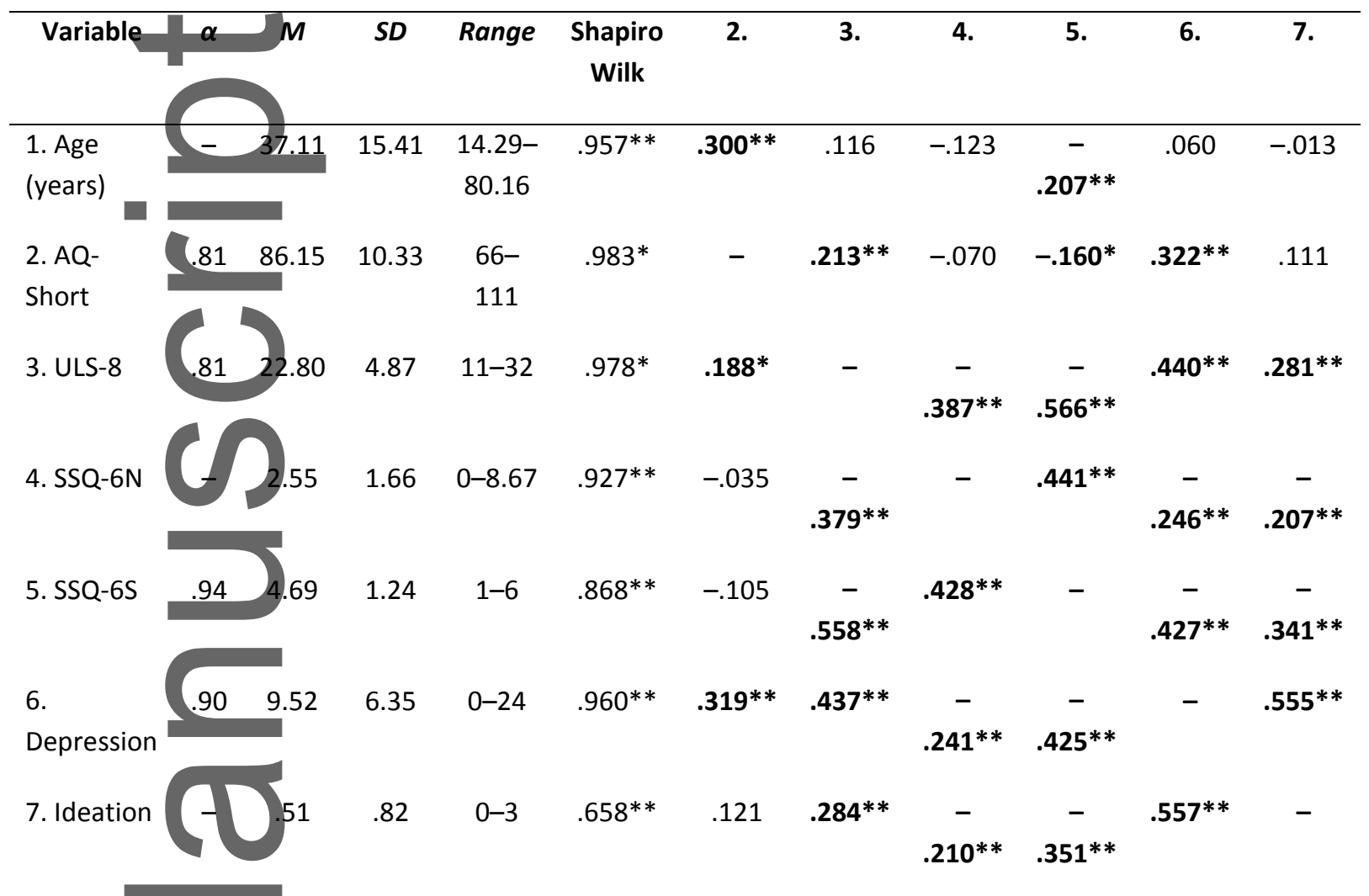

Notes. $\alpha=$ Cronbach's alpha. AQ-Short: Autism Spectrum Quotient-Short; ULS-8: University of California Los Angeles Loneliness Scale-Short Form; SSQ-6N: Social Support Questionnaire Short Number; SSQ-6S: Social Support Questionnaire Short Satisfaction

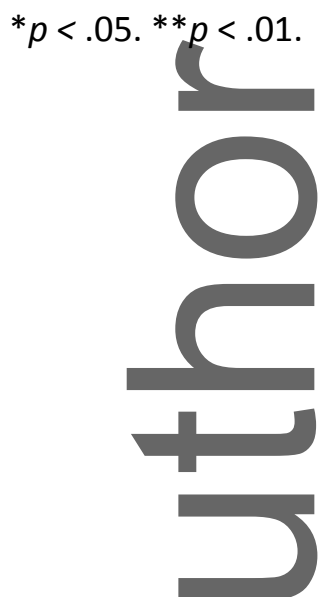

This is the roth r manuscript accepted for publication and has undergone full peer review but has not been through tho pyediting, typesetting, pagination and proofreading process, which may lead to differences between this version and the Version of Record. Please cite this article as doi: 10.1002/da.22759.

This article is protected by copyright. All rights reserved. 
Table 4. Descriptive $(M, S D)$ and bootstrapped between-group comparisons for study variables by gender (males, $n=83$; females, $n=92$ )

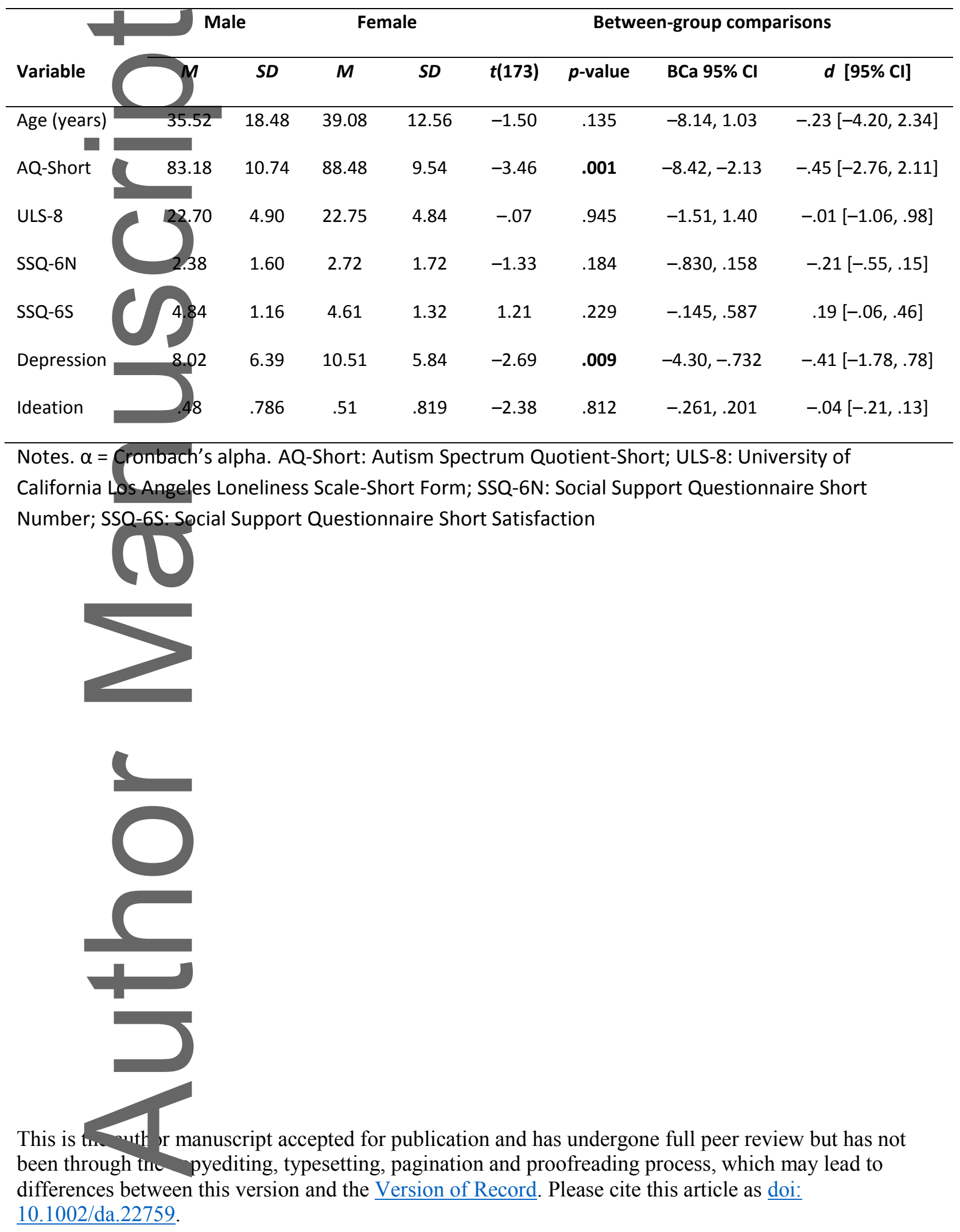

This article is protected by copyright. All rights reserved. 
Table 5. Pearson's bootstrapped partial correlations controlling for age between study variables for females (upper; $n=92$ ), and males (lower; $n=83$ )

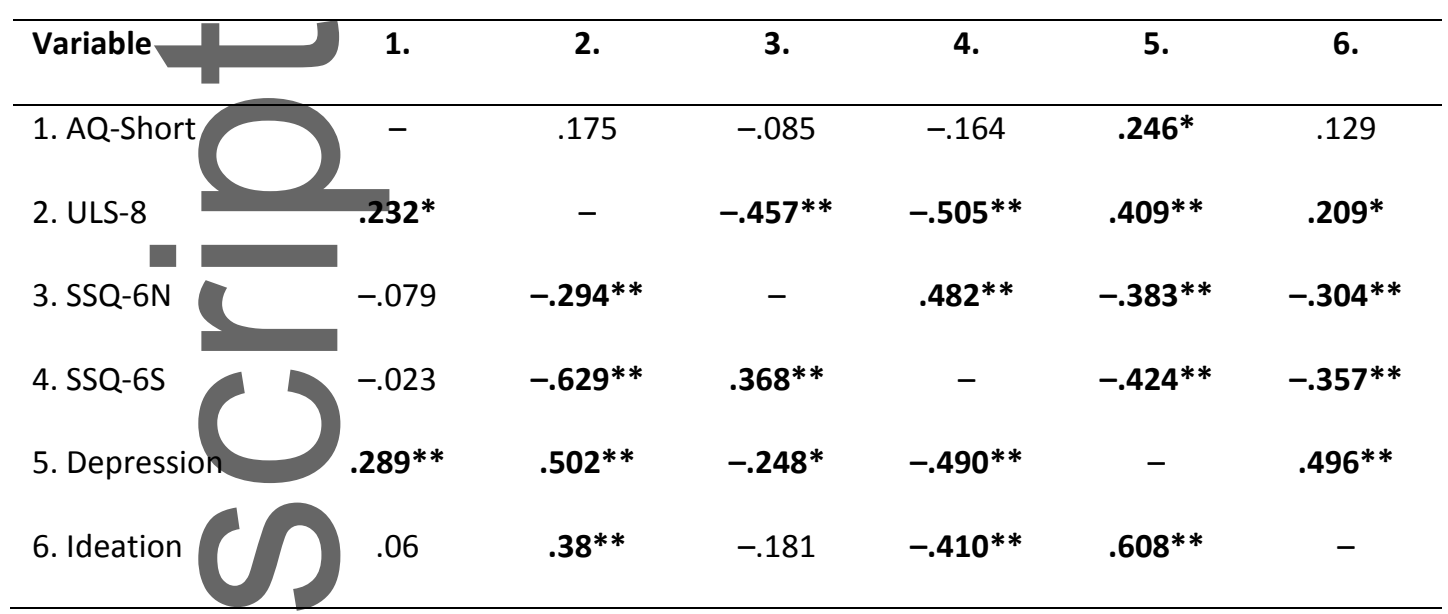

Notes. $\alpha=$ Cronbach's alpha. AQ-Short: Autism Spectrum Quotient-Short; ULS-8: University of California Los Angeles Loneliness Scale, Short Form; SSQ-6N: Social Support Questionnaire Number; SSQ-6S: Social Support Questionnaire Satisfaction

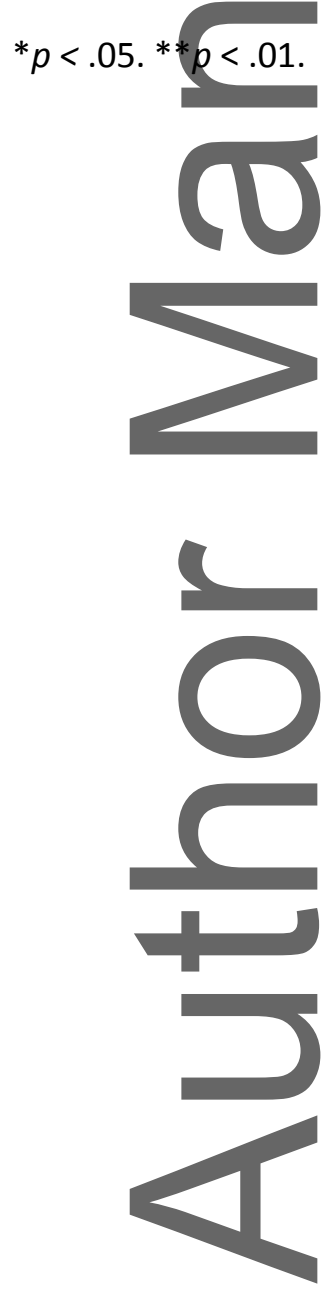

This article is protected by copyright. All rights reserved.

Corresponding author: Darren Hedley 
Table 6. Regression Models predicting PHQ Depression and Suicidal Ideation

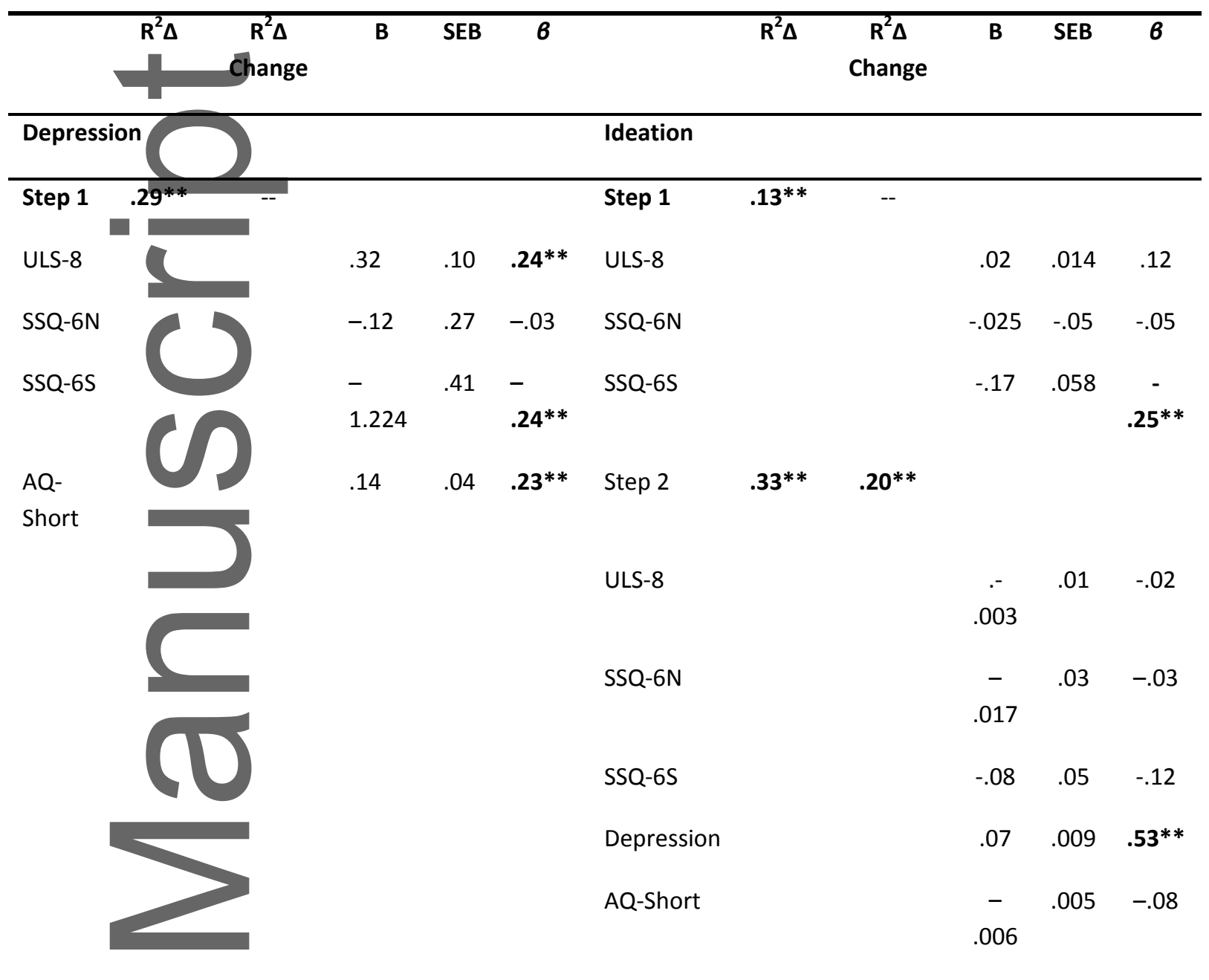

Note: AQ-Short: Autism Spectrum Quotient-Short; ULS-8: University of California Los Angeles Loneliness Scale-Short Form; SSQ-6N: Social Support Questionnaire Short Number; SSQ-6S: Social Support Questionnaire Short Satisfaction

$* p<.05 . * *<.01$

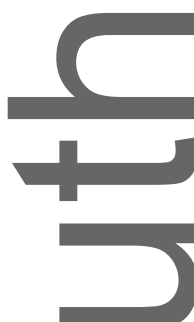

This is the roth r manuscript accepted for publication and has undergone full peer review but has not been through thu pyediting, typesetting, pagination and proofreading process, which may lead to differences between this version and the Version of Record. Please cite this article as doi: 10.1002/da.22759.

This article is protected by copyright. All rights reserved. 


\section{Figure legend}

Figure 1. Path model.

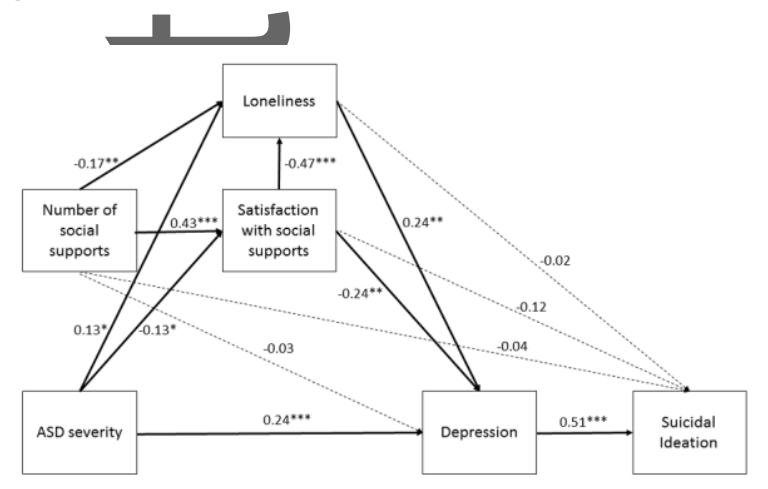

Figure 1. Path model.

${ }^{*} p<.05 ; * *_{p}<.01 ; * * * p<.001$
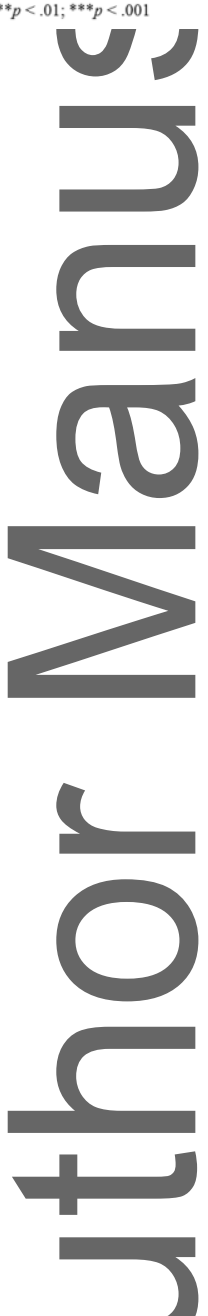

This is thenth r manuscript accepted for publication and has undergone full peer review but has not been through thu pyediting, typesetting, pagination and proofreading process, which may lead to differences between this version and the Version of Record. Please cite this article as doi: 10.1002/da.22759.

This article is protected by copyright. All rights reserved. 


\section{University Library}

\section{- M M N E R VA A gateway to Melbourne's research publications}

Minerva Access is the Institutional Repository of The University of Melbourne

Author/s:

Hedley, D;Uljarevic, M;Foley, K-R;Richdale, A;Trollor, J

Title:

Risk and protective factors underlying depression and suicidal ideation in Autism Spectrum Disorder

Date:

2018-07-01

Citation:

Hedley, D., Uljarevic, M., Foley, K. -R., Richdale, A. \& Trollor, J. (2018). Risk and protective factors underlying depression and suicidal ideation in Autism Spectrum Disorder. DEPRESSION AND ANXIETY, 35 (7), pp.648-657. https://doi.org/10.1002/da.22759.

Persistent Link:

http://hdl.handle.net/11343/284941 